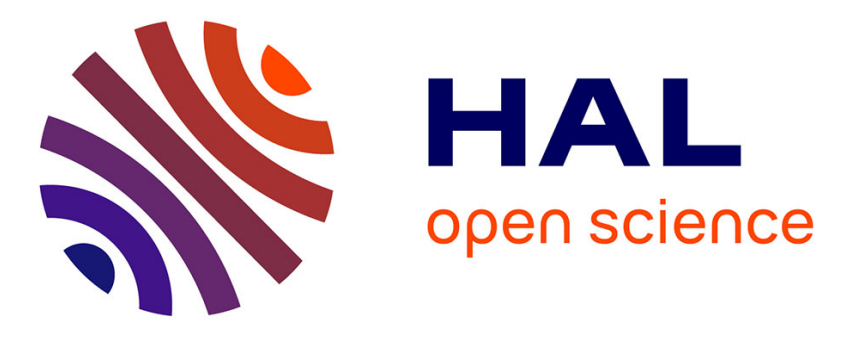

\title{
A multi-channel approach for cortical stimulation artefact suppression in depth EEG signals using time-frequency and spatial filtering
}

Abhijit Bhattacharyya, Radu Ranta, Steven Le Cam, Valérie Louis-Dorr, Louise Tyvaert, Sophie Colnat-Coulbois, Louis Maillard, Ram Bilas Pachori

\section{To cite this version:}

Abhijit Bhattacharyya, Radu Ranta, Steven Le Cam, Valérie Louis-Dorr, Louise Tyvaert, et al.. A multi-channel approach for cortical stimulation artefact suppression in depth EEG signals using time-frequency and spatial filtering. IEEE Transactions on Biomedical Engineering, 2019,66 (7), pp.1915-1926. 10.1109/TBME.2018.2881051 . hal-01917304

\author{
HAL Id: hal-01917304 \\ https://hal.science/hal-01917304
}

Submitted on 9 Nov 2018

HAL is a multi-disciplinary open access archive for the deposit and dissemination of scientific research documents, whether they are published or not. The documents may come from teaching and research institutions in France or abroad, or from public or private research centers.
L'archive ouverte pluridisciplinaire HAL, est destinée au dépôt et à la diffusion de documents scientifiques de niveau recherche, publiés ou non, émanant des établissements d'enseignement et de recherche français ou étrangers, des laboratoires publics ou privés. 


\title{
A multi-channel approach for cortical
}

\section{stimulation artefact suppression in depth EEG \\ signals using time-frequency and spatial \\ filtering}

\author{
A. Bhattacharyya, R. Ranta, S. Le Cam, V. Louis-Dorr, L. Tyvaert, \\ S. Colnat-Coulbois, L. Maillard and R. B. Pachori
}

\begin{abstract}
Objective: The stereo electroencephalogram (SEEG) recordings are the sate of the art tool used in pre-surgical evaluation of drug-unresponsive epileptic patients. Coupled with SEEG, electrical cortical stimulation (CS) offer a complementary tool to investigate the lesioned/healthy brain regions and to identify the epileptic zones with precision. However, the propagation of this stimulation inside the brain masks the cerebral activity recorded by nearby multi-contact SEEG electrodes. The objective of this paper is to propose a novel filtering approach for suppressing the CS artifact in SEEG signals using time, frequency as well as spatial information. Methods: The method combines spatial filtering with tunableQ wavelet transform (TQWT). SEEG signals are spatially filtered to isolate the CS artifacts within a few number of sources/components. The artifacted components are then decomposed into oscillatory background and sharp varying transient signals using tunable-Q wavelet transform (TQWT). The CS artifact is assumed to lie in the transient part of the signal. Using prior known time-frequency information of the CS artifacts, we selectively mask the wavelet coefficients of the transient signal and extract out any remaining significant electro-physiological activity. Results: We have applied our proposed method of CS artifact suppression on simulated and real SEEG signals with convincing performance. The experimental
\end{abstract}

A. Bhattacharyya (e-mail: abhijit9800@gmail.com) and R. B. Pachori are with Discipline of Electrical Engineering, Indian Institute of Technology Indore, Indore- 453552, India.

R. Ranta (e-mail: radu.ranta@univ-lorraine.fr), S. Le Cam, V. Louis-Dorr, L. Tyvaert, S. Colnat-Coulbois, L. Maillard are with Université de Lorraine, CNRS, CRAN, F-54000 Nancy, France.

L. Tyvaert, S. Colnat-Coulbois, L. Maillard are also with Université de Lorraine, CHRU-Nancy, Service de neurologie, F-54000, France 
results indicate the effectiveness of the proposed approach. Conclusion: The proposed method suppresses CS artifacts without affecting the background SEEG signal. Significance: The proposed method can be applied for suppressing both low and high frequency CS artifacts and outperforms current methods from the literature.

\section{Index Terms}

cortical stimulation, SEEG, tunable-Q wavelet transform, subspace correlation approach, blind source separation.

\section{INTRODUCTION}

The accurate localization of epileptiform discharges is considered to be very crucial in the diagnosis of patients undergoing resective epileptic surgery [1]. In this purpose, Stereo-electroencephalography (SEEG), performed using electrode shafts implanted within the brain volume, is considered as the gold standard [2], [3]. Apart from this purely clinical purpose, the SEEG signals can be also used to explore the complex brain responses in the presence of provoked electrical stimulations. In particular, the direct cortical stimulation (CS) is a decisive diagnostic measure for localizing epileptogenic zone and eloquent cortex in the pharmacoresistant epilepsy patients who undergo presurgical invasive investigations with depth electrodes [4]. Different stimulation techniques have been discussed, but in principle all of them are based on current injection between two neighboring contacts of an SEEG shaft. The high frequency CS (HFCS) (in the range of 50-55 Hz) has been used in both extraoperative and intraoperative CS [1]. On the other hand, low frequency CS (LFCS) (generally at $1 \mathrm{~Hz}$ ) is recommended for functional mapping of cortical areas. Both LFCS and HFCS are used to localize eloquent and epileptogenic zones. In most of the cases, no clinical signs are induced by LFCS. However, the LFCS has some possible advantages such as: 1. epileptogenic zone definition (LFCS may induce seizure in the epileptogenic zone especially hippocampus), 2. lower rate of after discharges [5], 3. motor cortical mapping (sometimes memory function mapping) 4. generates cortico-cortical evoked potentials (CCEP), which indicate direct or indirect interconnections between the recording and stimulated sites [6]. The CCEP have been found useful for clinical study of epileptic networks, for mapping functional networks which include motor control, language networks, intra and inter-lobar connectivity. 
From a measurement point of view, the intracerebral SEEG electrodes are roughly similar to surface EEG electrodes (they possess a large surface area, of the order of several $\mathrm{mm}^{2}$, thus they record electrical activities of rather large brain sources). The usual macroscopic approximation holds (dipolar current sources and linear instantaneous propagation of the electric field). Consequently, we consider that the recordings are a linear mixing of the CS artifact and of the physiological brain activity, either evoked by the CS or background. Thus, it is of high clinical interest to decouple the stimulation source from the brain sources so that recovered signals can be analyzed later.

There exist several methods for suppression of artifacts generated as a result of electrical stimulation. In [7] authors proposed Hampel filter based cancellation of stimulation artifacts. The Hampel identifier analyzed the frequency spectrum of the signal and replaced the outliers in the frequency domain with interpolated values. Finally, they recovered the clean signal after inverse Fourier transfom. In [8], stimulation artifacts were suppressed using frequential filtering and a generalized eigenvalue decomposition (GEVD) based method for spatial filtering. In [6], the authors proposed a method for identifying and next canceling stimulation artifacts based on linear regression of the measured signals on simulated models of the CS artifact including models of the tissue-electrode interface.

The previous methods are mostly mono-channel approach for CS artifact suppression. Recently, multicomponent multivariate signal decomposition techniques have gained lot of attention [9], [10]. In particular, empirical mode decomposition (EMD) [11] and synchrosqueezed wavelet transform [12] have been extended for multivariate signals [13]-[15], providing more accurate estimation of components shared among the channels, and proved to be successful in various applications [16]-[18].

Another important aspect in the context of CS artifact cancellation is that prior information on the nature of the components to separate is available. Indeed EEG signal exhibits a certain degree of oscillatory nature, as opposed to the transient aspect of the CS artifact to be removed. A proper tuning of the decomposition bases adapted to the oscillatory nature of the signal can be achieved by the use of tunable-Q wavelet transform (TQWT) [19], [20], a wavelet transform in which the Q-factor can be easily and continuously tuned. In literature, TQWT has been used extensively for analysis of nonstationary signals [21], [22]. By choosing two different Q-factors adapted to the oscillatory and transient parts of the signal respectively, the sparsity of the decomposition will be increased [20] and provide better separation between these two 
signal components. In this work, we propose two approaches for suppressing CS artifacts from SEEG signals using a combination of TQWT and different spatial filtering methods. In the first approach, non-linear TQWT based filtering of each individual SEEG channel is applied. The TQWT sparsely represents a CS artifact affected channel as the sum of non-oscillatory transients (mostly CS artifacts) and oscillatory (background EEG) signals using low and high quality factors (Q) simultaneously. Then, we use prior known time-frequency information of the CS artifacts for extracting the propagated CS artifact pattern from the transient signal without presence of any electro-physiological activity. In the second approach, we propose to enhance our proposed TQWT based filtering method by combining it with different spatial filtering methods, inspired either from independent component analysis (e.g., [23]) or from subspace analysis techniques [24]. This has been carried out by applying these spatial filtering techniques to multi-channel SEEG signals in order to isolate and enhance the CS artifacts within few components/sources in the transformed domain. Then, the previously described TQWT based filtering is applied on those few artifact dominated components only, before reconstructing the clean SEEG signals with an inverse spatial filtering operation. In this work, we present the performance of the proposed methods in suppressing both low and high frequency CS artifacts.

The rest of the paper is organized as follows: Section II describes the generation of multichannel simulated SEEG signals and real SEEG signals used in this work. Section III presents the proposed framework of multichannel CS artefact suppression method, Section IV presents the experimental outcome and discusses the effectiveness of the proposed method. Finally, Section $\mathrm{V}$ concludes the paper.

\section{Simulated AND REAL DATASETS}

\section{A. Simulated datasets}

The performance of the proposed method has been analyzed on synthetically generated realistic SEEG datasets. To this purpose, we have simulated realistic CS, CCEP and background sources, propagating in a realistic, SEEG implanted, head model.

We have used the adult MNI-ICBM152 averaged model, available for download on the web [25], [26]. Dipolar sources are placed on a regular 3D grid with 9mm distance between each dipole position, within the segmented gray matter. The resulting 523 dipole positions are next considered as sources of physiological activity. Then, the simulated SEEG electrodes are placed in realistic way following a plausible clinical implantation scheme used in temporal lobe 
epilepsy context (see Fig. 1). To be more precise, 3 simulated multielectrodes are placed in the left hemisphere, whereas 9 in the right hemisphere. It should be noted that each of these multielectrodes have 7 to 10 sensors inside the brain. The total number of sensors placed inside the brain is 186, 47 sensors being placed in the left hemisphere and 139 in the right. A five compartiment finite element modelling (FEM) is used to compute realistic lead-field (LF) matrix which represents the volume conduction forward model. For a more detailed description of the simulation setup, see [27]. As for every position we can have 3 elementary orthogonal dipoles orientated as, Ax: inion-nasion, Ay: right-left and Az: bottom-up, the final dimension of the LF matrix is $186 \times 1569$ (number of channels by number of elementary dipoles).

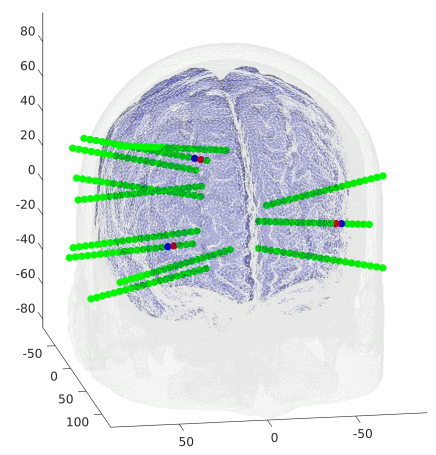

Fig. 1: Plot of depth electrode positions (in green) and considered dipole sources (in red and blue) in the brain volume.

The recorded signals were then simulated as a linear mixture of three types of sources: the CS dipole source, the CCEP source (optionally), and background physiological sources.

a) CS dipole source: among the 523 source positions, we have chosen three of them, between neighboring contacts of three different shafts. They were considered as the positions of the CS dipole. These three positions are deep inside the brain, intermediate and superficial. For a particular experimental session, one of these three positions is chosen as the active CS source. The orientation of the CS dipole was set along the corresponding SEEG shaft, as in real clinical situations (recall that the current is injected between two neighboring contacts of a shaft). The time varying amplitude of the CS was generated as a very short duration (1.05 ms) biphasic 
current pulse at a very high sampling rate:

$$
p(t)=\left\{\begin{aligned}
-A, & t \in\left[-\left(T_{p}+t_{p}\right), \quad-t_{p}\right] \\
A, & t \in\left[t_{p}, \quad T_{p}+t_{p}\right] \\
0, & \text { elsewhere }
\end{aligned}\right.
$$

with $A=0.5-3 \mathrm{~mA}, T_{p}=0.5 \mathrm{~ms}$ and $t_{p}=0.025 \mathrm{~ms}$.

The CS pattern is next passed through a high-pass filter (cutoff frequency $0.15 \mathrm{~Hz}$ ) and an anti-aliasing filter before down-sampling at $512 \mathrm{~Hz}$ (in order to mimic the clinically used acquisition system, see below subsection II-B). We have generated both type of stimulation sessions namely LFCS (pattern frequency at $1 \mathrm{~Hz}$ ) and HFCS (pattern frequency at $55 \mathrm{~Hz}$ ). The detailed description related to time and frequency analysis for this type of model of current stimulation is presented in [8], [28].

b) CCEP source: The CCEP source position is selected as the nearest position to the CS dipole source. The orientation was randomly generated, while the time varying amplitude was simulated by multiplying a slow varying sinusoidal wave with a decaying exponential. Note that the CCEP source is considered only for the LFCS session. For HFCS, we have not considered the CCEP source, as its latency is above the HFCS period.

c) Background physiological sources: The remaining 521 positions are responsible for generating background physiological activity. The orientation were random, while the time courses were generated using the nonstationary EEG model developed in [29] and adapted for SEEG in [30].

Overall, $C=186$ signals of length $N$ were simulated as:

$$
\mathbf{X}=\mathbf{L F}_{C S} \mathbf{s}_{C S}+\mathbf{L} \mathbf{F}_{C C E P} \mathbf{s}_{C C E P}+\mathbf{L} \mathbf{F}_{B} \mathbf{s}_{B}
$$

with sources $\mathbf{s}_{C S}, \mathbf{s}_{C C E P} \in \mathbb{R}^{3 \times N}$ and $\mathbf{s}_{B} \in \mathbb{R}^{1563 \times N}$, and lead-field matrices $\mathbf{L F} \in \mathbb{R}^{C \times N_{s}}$, with $N_{s}$ being 3, 3 and 1563 respectively for the three types of sources.

Finally, we have simulated the effects of acquisition instrument such as saturation and jitter. It should be noted that the electrodes close to the stimulation site face saturation effect due to the fact that amplitudes of the propagated stimulations exceed the acquisition system range. For making the session realistic, we have considered random sampling jitters of maximum 5 samples across all the 186 channels which yields a slight desynchronization of CS artifacts across the channels. 
This procedure simulates one complete session of multichannel synthetic SEEG signals subjected to CS. We have generated 50 such different stimulation sessions for each of the stimulation type (LFCS and HFCS) by changing the CS source location across the three different mentioned brain locations and considering different SEEG background sources. Fig. 2 presents an example of synthetically generated SEEG signals affected with LFCS artefact. A similar figure can be generated for simulated session subjected to HFCS also.

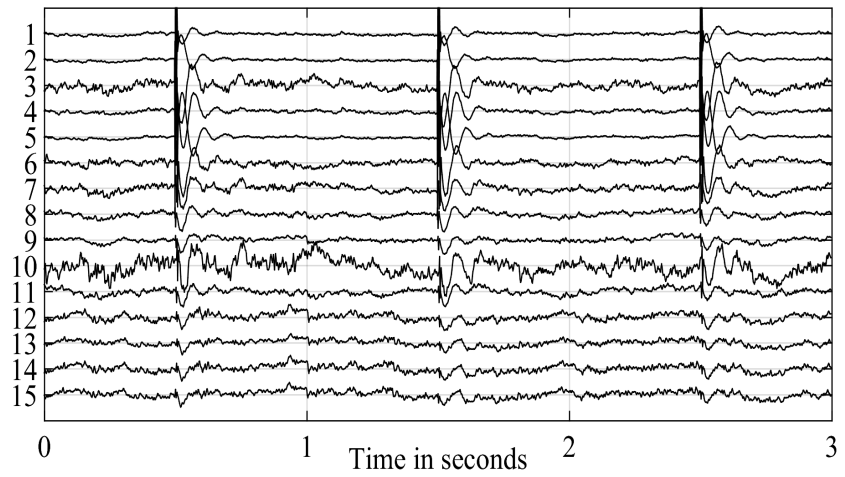

Fig. 2: Synthetically generated SEEG signals during LFCS with $1 \mathrm{~mA}$ biphasic current pattern.

\section{B. Real datasets}

In order to validate the performance of the proposed method we have used real SEEG datasets affected by CS artifacts, recorded in clinical routine at the Nancy University Hospital (CHU Nancy), France. The procedure was approved by the ethics committee of the Nancy Hospital and the patients gave their informed consent. Both types of stimulation were analyzed, namely LFCS and HFCS. For each type of stimulation, we have used 50 different sessions recorded from 4 patients. In case of HFCS the signals were recorded with sampling frequency of $512 \mathrm{~Hz}$, where as for LFCS the signals had two different sampling frequencies as $1024 \mathrm{~Hz}$ and $2048 \mathrm{~Hz}$. An example of LFCS recording is presented Fig. 3.

\section{METHODS}

\section{A. Tunable- $Q$ wavelet transform}

The TQWT is implemented with the idea of two channel filter bank structure. Let $\zeta$ and $\kappa$ denote the high-pass and low-pass scale factors, respectively, of the two channel filter bank. The 


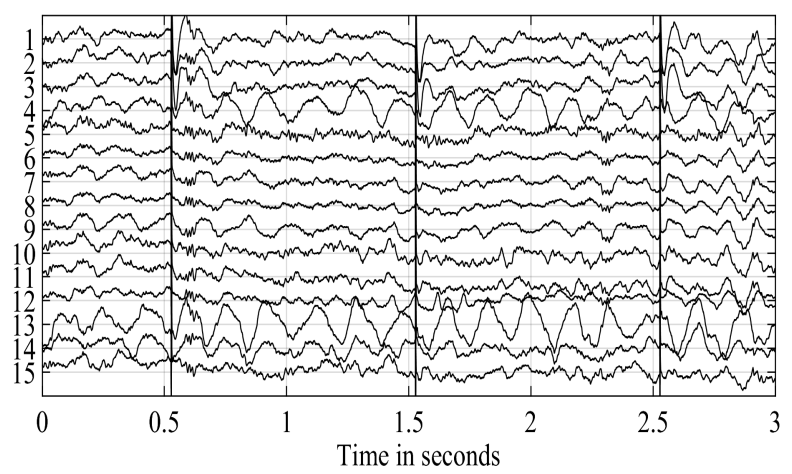

Fig. 3: Real SEEG signals recorded during LFCS with $1 \mathrm{~mA}$ biphasic current pattern.

low-pass filter frequency response in TQWT is mathematically defined as:

$$
\Lambda_{0}(\Omega)= \begin{cases}1, & \text { if }|\Omega| \leq(1-\zeta) \pi \\ \Psi\left(\frac{\Omega+(\zeta-1) \pi}{\kappa+\zeta-1}\right), & \text { if }(1-\zeta) \pi<|\Omega|<\kappa \pi \\ 0, & \text { if } \kappa \pi \leq|\Omega| \leq \pi\end{cases}
$$

while the high-pass filter frequency response writes [19]:

$$
\Lambda_{1}(\Omega)= \begin{cases}0, & \text { if }|\Omega| \leq(1-\zeta) \pi, \\ \Psi\left(\frac{\kappa \pi-\Omega}{\kappa+\zeta-1}\right), & \text { if }(1-\zeta) \pi<|\Omega|<\kappa \pi \\ 1, & \text { if } \kappa \pi \leq|\Omega| \leq \pi,\end{cases}
$$

where $\Psi(\Omega)$ denotes Daubechies filter frequency response [19]. The variables $\kappa$ and $\zeta$ are chosen so that they obey the following conditions

$$
0<\kappa<1 ; \quad 0<\zeta \leq 1 ; \quad \kappa+\zeta>1
$$

The quality factor $(Q)$, maximum number of sub-bands $\left(J_{\max }\right)$, and redundancy parameter $(R)$ of TQWT can be expressed in terms of low-pass and high-pass scale factors as follows: [19]:

$$
Q=\frac{2-\zeta}{\zeta} ; \quad J_{\max }=\left[\frac{\log (\zeta N / 8)}{\log (1 / \kappa)}\right] ; \quad R=\frac{\zeta}{1-\kappa},
$$

where analyzed signal length is denoted by $N$.

The wavelets generated for different $Q$-factors are shown in Fig. 4. It can be seen that, for $Q=1$ the generated wavelet has transient shape without any oscillatory behavior. On the other hand, increasing the value of $Q$ factor increases the number of oscillations in the 


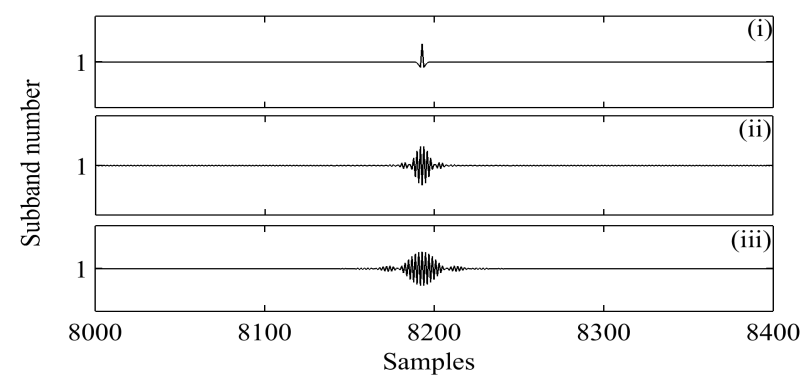

Fig. 4: Plots of wavelets corresponding to different $Q$ factors: (i) $Q=1, R=3$ (ii)

$$
Q=10, R=3 \text { (iii) } Q=20, R=3
$$

mother wavelet (see Fig. 4 for $Q=20$ ). Thus, it is advised to select low $Q$ factors for sparsely representing transient signals and high $Q$ factor for sparse representation of oscillatory signals. The redundancy parameter $R$ has the role of localizing the wavelet in time domain without affecting its shape. In the frequency domain, more overlapping is observed between adjacent frequency bands with increased value of $R$, while $Q$ remains fixed. Thus, it is necessary to put more number of levels to cover the entire frequency range.

However, in real life, sparse representation of some signals may not be possible with a single $Q$-factor [20]. In such case, the concept of dual $Q$-factor can be utilized [20]: let the analyzed signal $x$ be represented as $x=x_{1}+x_{2}$, and let $x_{1}$ and $x_{2}$ have sparse representations using transforms $\Upsilon_{1}$ and $\Upsilon_{2}$ respectively. This sparse representation can be achieved following a basis pursuit approach, using $L_{1}$-norm minimization. The optimization problem is expressed as [20]:

$$
\begin{array}{ll}
\underset{\mathbf{w}_{\mathbf{1}}, \mathbf{w}_{\mathbf{2}}}{\operatorname{minimize}} & \left\|\mathbf{s}_{\mathbf{1}} \mathbf{w}_{\mathbf{1}}\right\|_{\mathbf{1}}+\left\|\mathbf{s}_{\mathbf{2}} \mathbf{w}_{\mathbf{2}}\right\|_{\mathbf{1}} \\
\text { subject to } & x=\Upsilon_{1}^{*} \mathbf{w}_{\mathbf{1}}+\Upsilon_{2}^{*} \mathbf{w}_{\mathbf{2}} .
\end{array}
$$

where $\Upsilon_{1}{ }^{*}$ and $\Upsilon_{2}{ }^{*}$ denote the inverse TQWT operations applied on sparse set of wavelet coefficients $\mathbf{w}_{\mathbf{1}}$ and $\mathbf{w}_{\mathbf{2}}$, respectively. In eqn (7), $\mathbf{s}_{\mathbf{1}}$ and $\mathbf{s}_{\mathbf{2}}$ are the weighting parameters expressed as

$$
\mathrm{s}_{1}=\Phi_{1}\left\|\Psi_{1}(\mathrm{k})\right\|_{2}, \quad \mathrm{~s}_{2}=\Phi_{2}\left\|\Psi_{2}(\mathrm{k})\right\|_{2}
$$

with $\Psi_{1}(\mathrm{k})$ and $\Psi_{\mathbf{2}}(\mathrm{k})$ being the wavelets corresponding to the wavelet coefficients $\mathbf{w}_{\mathbf{1}}(k)$ and $\mathbf{w}_{\mathbf{2}}(k)$, respectively. The parameters $\boldsymbol{\Phi}_{1}$ and $\boldsymbol{\Phi}_{\mathbf{2}}$ are used to calibrate the relative energy of estimated signal components $x_{1}$ and $x_{2}$, respectively [20]. 
The optimization problem of equation (7) is solved using an iterative algorithm known as split augmented Lagrangian shrinkage algorithm (SALSA) [31].

Finally, the signal components $x_{1}$ and $x_{2}$ can be estimated by inverse TQWT operation as:

$$
x_{1}=\Upsilon_{\mathbf{1}}{ }^{*} \mathbf{w}_{\mathbf{1}} \text { and } x_{2}=\Upsilon_{\mathbf{2}}{ }^{*} \mathbf{w}_{\mathbf{2}}
$$

Note that, before the reconstruction of the signal $x=x_{1}+x_{2}$ (8), one can also perform nonlinear operations such as thresholding or masking of certain wavelet coefficients. This operation, which can be seen as denoising, can either be implemented directly within the optimization problem by not requiring exact reconstruction [32] or by using prior knowledge on the timefrequency localization of the components of interest (transients/oscillatory, see also our proposed pipeline, subsection III-C).

\section{B. Spatial filtering}

In this context of multichannel recording setup formalized as a linear mixture (2), spatial filtering approaches can complement the monochannel TQWT methodology introduced in the previous section. These approaches are frequently used for EEG artifact canceling (see e.g., [33]), but also for CS/DBS artifact elimination [8], as they isolate and enhance the artifacts on one or few components. The signal space is separated in subspaces specific to different types of activities, in our case CS artifacts and physiological sources, and then only the physiological subspace is used to reconstruct the cleaned signal. We briefly recall below some of the most widely used spatial filtering techniques in the EEG artifact canceling context and proposes a new filtering approach, inspired from subspace correlation techniques [24], [34].

The first common step is to find a basis of the (complete) signal subspace, usually by whitening. If $\mathbf{X}$ are the recorded signals ( $N \times C$, samples by channels), then this basis $\mathbf{Z}$ can be found after eigendecomposition of the covariance/scatter matrix $\mathbf{R}_{\mathbf{x}}=\mathbf{X}^{T} \mathbf{X}=\mathbf{U D U}^{T}$ :

$$
\mathrm{Z}=\mathbf{X U D}^{-\frac{1}{2}}
$$

Clearly, right multiplying $\mathbf{Z}$ by any orthogonal matrix $\mathbf{J}$ yields another basis of the same signal space. The second step is then to find a specific orthogonal matrix $\mathbf{J}$, optimized for a specific criterion. In our case, the aim is to rotate the $\mathbf{Z}$ basis such as the artifact and physiological subspaces become as orthogonal as possible between them (i.e., some of the basis vectors/sources/components contain the artifacts, while the complementary basis vectors contain the physiological sources). 
1) Blind source separation: Following the hypothesis that the CS artifact pattern is independent of brain sources and projected linearly onto the sensors, any BSS algorithm may be utilized in order to extract the CS sources, without any knowledge of the mixing coefficients. In other words, even if the volume conduction model (LF matrix) is unknown or uncertain, one can still separate between artifacts and physiological activities, if the linear mixture and the independence between them (or multiple decorrelation, see below) hypothesis is respected.

Several families of BSS algorithms were proposed in the literature (see e.g., [23], [35] for details). The most well known algorithms are based either on Independent Component Analysis $(\text { ICA })^{1}$ [36]-[38], on second order statistics only (simultaneous diagonalization) [39], [40] or on combinations between them [41], [42].

2) Filtering/GEVD: The basic idea proposed in [8] is the following: applying a low-pass filter on the measured data $\mathbf{X}$ (channel by channel) will eliminate most of the CS artifact (very brief and thus large-band) and will provide a second data set $\mathbf{X}_{2}$. Next, this second data set can be transformed to

$$
\mathbf{Z}_{2}=\mathbf{X}_{2} \mathbf{U D}^{-\frac{1}{2}}
$$

with $\mathbf{U}$ and $\mathbf{D}$ the eigenvectors and eigenvalues of the first data set (9). Its covariance matrix can be diagonalized by eigendecomposition also, yielding $\mathbf{R}_{\mathbf{z}, 2}=\mathbf{Z}_{2}^{T} \mathbf{Z}_{2}=\mathbf{U}_{2} \mathbf{D}_{2} \mathbf{U}_{2}^{T}$. Finally, the desired rotation matrix will be $\mathbf{J}=\mathbf{U}_{2}$ and the complete spatial filter

$$
\mathbf{V}=\mathbf{U D}^{-\frac{1}{2}} \mathbf{U}_{2}
$$

One can recognize the joint diagonalization of the two data sets covariance matrices $\mathbf{R}_{\mathrm{x}}$ and $\mathbf{R}_{\mathbf{x}, 2}$ by GEVD:

$$
\left\{\begin{array}{c}
\mathbf{V}^{T} \mathbf{R}_{\mathbf{x}, 2} \mathbf{V}=\mathbf{D}_{2} \\
\mathbf{V}^{T} \mathbf{R}_{\mathbf{x}} \mathbf{V}=\mathbf{I}
\end{array}\right.
$$

The spatially filtered signals $\mathbf{Y}=\mathrm{XV}$ will naturally be ordered starting from the common sources of the two data sets (i.e., low frequency physiological sources), while the CS artifact sources will appear among the last components.

Different low-pass filters were tested in the cited publication, with rather small differences (from singular spectrum analysis [43] to simple finite impulse response FIR filters). It is important to note though that this approach was only tested on HFCS signals (periodic by nature), for which

\footnotetext{
${ }^{1}$ Involving higher order statistics or information theoretical measures estimated from the signals.
} 
the spectral characteristics are rather different than for LFCS (see spectral analysis in [8] and [28], respectively).

3) Subspace correlation approach (SCA): A third approach for spatially separating source of CS artifacts and physiological activity is based on a somehow similar idea. More precisely, one can construct a second data set by simulating the CS artifact. Different versions of this artifact can be obtained by including the variability due to the characteristics of the acquisition system (possible electrode impedances [6], sampling jitter, delays, ... ). Let this second data set be Q. A basis for this dictionary space can be obtained by whitening:

$$
\mathrm{Z}_{\mathbf{q}}=\mathrm{QU}_{\mathbf{q}} \mathrm{D}_{\mathbf{q}}^{-1 / 2}
$$

Next, a common subspace between the dictionary $\mathbf{Q}$ and the data $\mathbf{X}$ can be obtained by rotating the two basis $\mathbf{Z}$ and $\mathbf{Z}_{\mathbf{q}}$ one onto the other, following the approach proposed in [24]:

1) perform the singular value decomposition (SVD) of $\mathbf{C}=\mathbf{Z}^{T} \mathbf{Z}_{\mathbf{q}}$

$$
\mathbf{C}=\mathbf{F} \Sigma \mathbf{G}^{T}
$$

2) take $\mathbf{F}$ as the desired rotation matrix and construct the spatial filter

$$
\mathbf{V}=\mathbf{U D}^{-\frac{1}{2}} \mathbf{F}
$$

(note that a second spatial filter can be constructed for the dictionary: $\mathbf{V}_{\mathbf{q}}=\mathbf{U q D q} \mathbf{q}^{-\frac{1}{2}} \mathbf{G}$; the two data sets and the two basis do not need to have the same dimension).

One can show that the two basis (signal space and dictionary space)

$$
\begin{aligned}
\mathbf{Y} & =\mathrm{XV} \\
\mathbf{Y}_{\mathbf{q}} & =\mathrm{QV}_{\mathbf{q}}
\end{aligned}
$$

are now aligned:

$$
\mathbf{Y}^{T} \mathbf{Y}_{\mathbf{q}}=\mathbf{\Sigma}
$$

The singular values close to 1 in $\Sigma$ indicate the common subspace basis between the dictionary and the data set [24]. Consequently, one can reconstruct the artifactual component of the original data set by simply using the common basis vectors (i.e., the artifact sources) from $\mathbf{Y}$ and projecting them back onto the signal space, the cleaned version being obtained by subtraction (or by using the complementary basis vectors). It should be noted that SCA yields a spatial filter that optimally decomposes the multichannel artifacted SEEG signal in "sources" close 
(correlated) to the dictionary (CS artefacts) and a complementary uncorrelated part (it is similar to the iteratively constructed canonical correlation analysis (CCA) [34]). The objective, and the obtained spatial filter, are thus different from the common spatial patterns (CSP) [17], [44] and its recently introduced version augmented complex CSP [45], which enhance the separability of two groups of signals by maximizing the variance of signals in one group while simultaneously minimizing the variance of signals belonging to the other group.

\section{CS artifact suppression pipeline}

Both TQWT and spatial filtering are in principle able to separate between CS artifacts and physiological signals. The present section proposes an original methodology for combining the two techniques. The rationale is straightforward: in order to facilitate the wavelet processing (either sparsity enhancement by SALSA or thresholding and masking), one can use spatial filtering as a preprocessing step aiming to separate the transients to be detected on fewer sources, enhancing them thus on these components and reducing the redundancy in the data. Moreover, this spatial preprocessing will in principle also ease the computational burden, as fewer signals will need to be processed by TQWT (only the artifact affected sources instead of potentially all measured channels).

Of course, different parameters need to be defined for our application and adapted depending on the type of stimulation (LFCS or HFCS). They are presented below.

1) TQWT parameters: All along this work, we have fixed $Q=1$ to define the wavelet basis corresponding to the CS artifact (transient) component of the SEEG signal, irrespective of the stimulation frequency. As already mentioned, the reason is very similar time frequency characteristics of the generated wavelet and of the CS transient (time support, but also Fourier spectrum). The $Q$-factor corresponding to oscillatory components of the signal can be chosen depending on the oscillatory nature of the signal. We have tested several values and achieved best performance when the high $Q$-factor is fixed to 20 and 10 for LFCS and HFCS, respectively. The redundancy parameter $(R)$ of TQWT is set to 3 throughout this work. The number of levels of TQWT corresponding to low and high $Q$-factor are set to maximum possible values following equation (6).

2) Non-linear processing : In real applications, in the process of dual $Q$-factor decomposition, the separation is not perfect and the extracted "transient" components of the real SEEG signals might also include significant physiological activity. Thus, an additional processing step is 
required to recover the slow varying wave from the transient component. This is performed with prior known time-frequency information of the CS artifacts.

More precisely, we have analyzed the wavelet coefficients corresponding to low $Q$-factor component. Let the number of oscillatory levels be $J_{1}$. It should be noted that the wavelet coefficients of first few higher oscillatory levels give rise to CS artifacts. At the same time, the time localization of the CS artifacts is also known to us as they appear periodically (every second or every $\sim 20 \mathrm{~ms}$ for LFCS and HFCS respectively). Thus, we can selectively mask the wavelet coefficients of first $L$ oscillatory levels which lie within specific small time windows (16ms) centered around the CS artifacts using the prior known time localization information.

Note that, in case of HFCS, we did not use the time localization information for masking the CS artifacts, as they appear much more frequently. Thus, for high frequency stimulation, all the wavelet coefficients (corresponding to transient component) which belong from first $L$ higher oscillatory levels were forced to zero.

This masking procedure allows us to extract separately the slow varying part remaining in the transient signal (low- $Q$ component) using an inverse TQWT operation and add it back to the oscillatory background.

The value of $L$ has been fixed to 13 and 4 for signals subject to LFCS and HFCS, respectively, after analyzing the frequency spectrum of TQWT sub-bands of higher oscillatory levels which give rise to the CS artifacts.

In Fig 5, we have presented the single channel real SEEG signal subjected to LFCS, its artifact free version, and the extracted LFCS pattern obtained with TQWT and nonlinear processing. It can be seen that the shapes of the CCEP responses (confined in ellipse) following the CS artifacts are not distorted and at the same time the artefact pattern is free from any background signal. Thus, the proposed method cancels the LFCS artifacts without distorting the background signal.

Fig. 6 presents the single channel real SEEG signal subjected to HFCS, the clean SEEG signal, and HFCS artifact pattern after applying TQWT and nonlinear processing. It is clear that the corrected signal is almost free from CS artifacts.

3) Multichannel CS artefact suppression: As mentioned, we have used spatial filtering to preprocess the data before TQWT. We have evaluated different flavors of BSS and subspace correlation approaches (SCA).

For the BSS case, we have tested several available algorithms (SOBI, FastICA, InfoMax, 


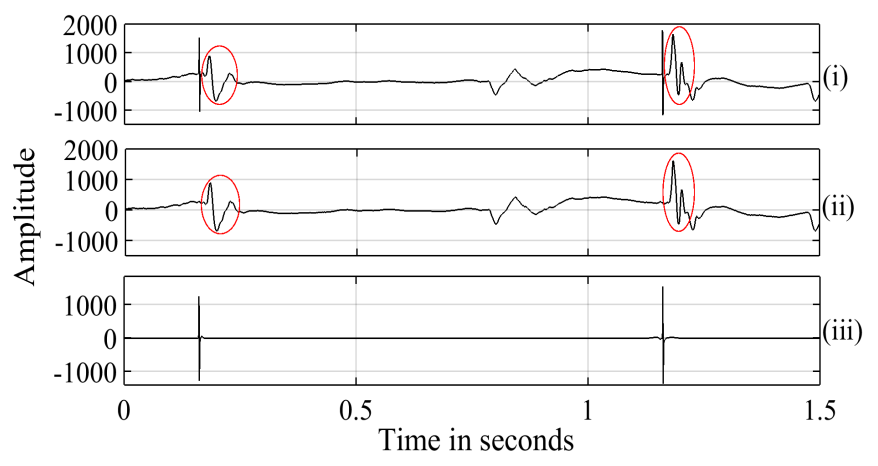

Fig. 5: Plots of (i) monochannel real SEEG signal subjected to LFCS, (iii) artefact free real SEEG signal using TQWT and nonlinear processing (ii) extracted LFCS artefact pattern. The CCEP responses followed by CS artifacts are encircled with ellipses.

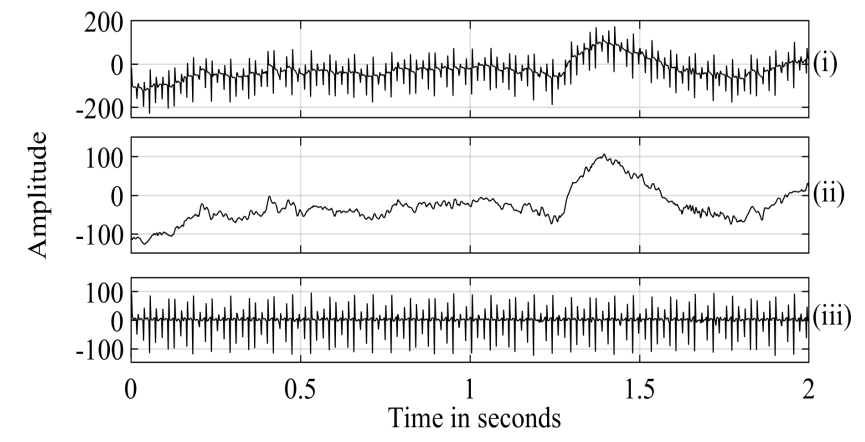

Fig. 6: Plots of real SEEG signal affected with HFCS artifacts (i), (ii) artefact free version of the real SEEG signal using TQWT and nonlinear processing (iii) extracted HFCS artefact pattern

COMBI, EFICA, WASOBI). Only the best results will be presented next. The results are different of course depending on the particular employed BSS algorithm or the dictionary construction in the subspace correlation method. Besides, a critical issue common to all methods is the selection of the artifacted components, on which TQWT needs to be applied. It is interesting to notice that the CS artifact appeared on several independent components, most certainly because of nonlinear effects (saturation, delays, jitter) and possibly non-independence (when CCEP are present in the data).

For the SCA, we have constructed the dictionary by convolving the CS pattern (1) successively 


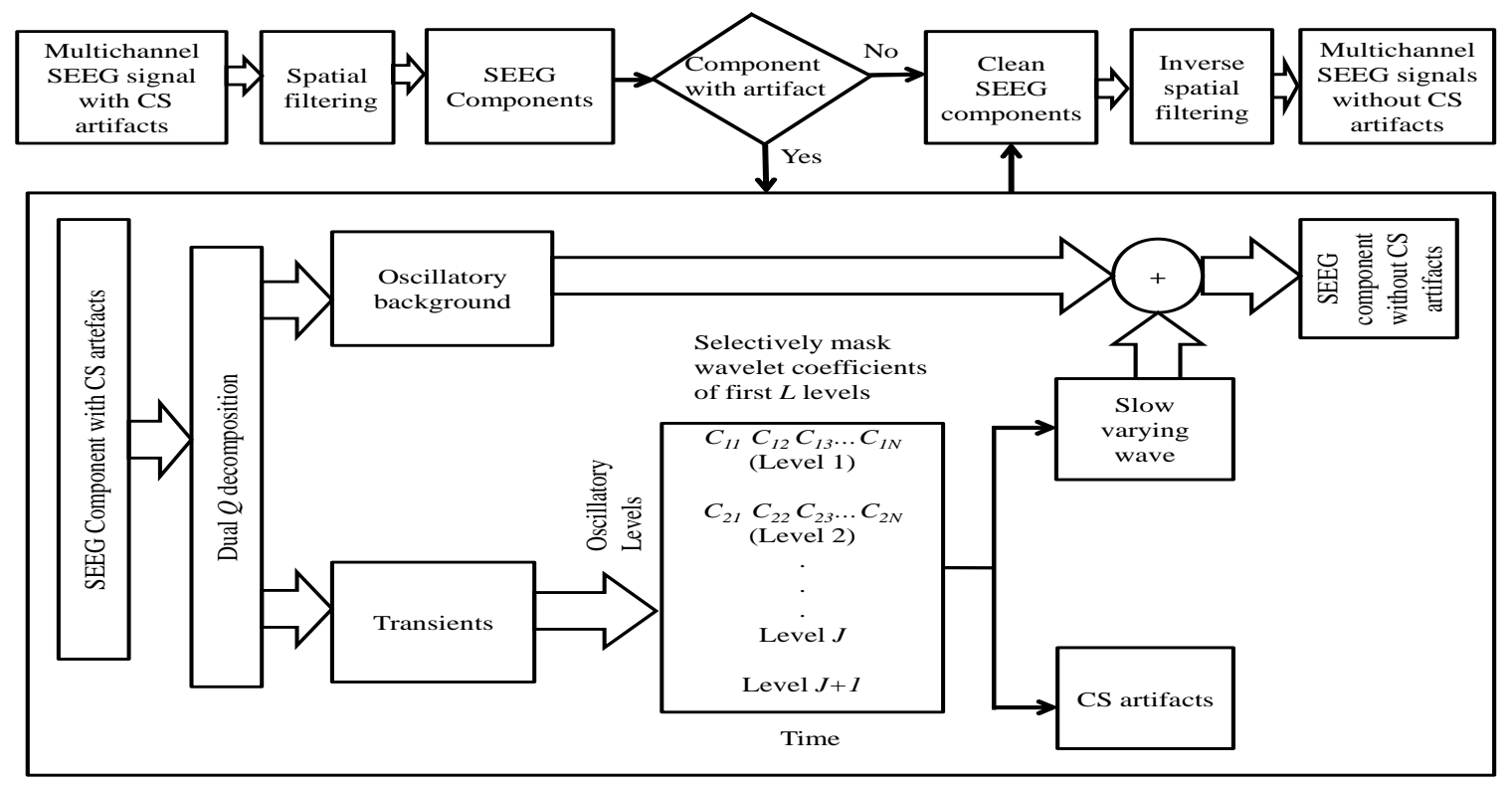

Fig. 7: Block diagram of the proposed multichannel channel CS artefact suppression method based on TQWT and spatial filtering

by the electrode-tissue interface (with several cut-off frequencies as indicated in [6]) and with the instrumentation acquisition filters (see above subsection II-A). The obtained signals (sampled initially at a very high sampling frequency), were down-sampled to the acquisition system sampling rate. In order to increase the variability of the dictionary and to cover possible instrumentation effects, we have simulated sampling jitter and buffering delays of up to 5 samples.

It should be noted that for SCA method the artifact sources (i.e., correlated with the dictionary subspace) appear in order. However, for BSS methods, we have selected the artifact sources as the ones having high correlation coefficients (defined in the next subsection, eqn (14)) with the simulated CS pattern. For all the considered spatial filtering methods, the artifact space dimension (number of components/sources with artifacts) were also visually inspected. The overall methodology is presented in Fig. 7.

\section{Performance evaluation}

For synthetic multichannel SEEG dataset, we know the ground truth signals. Hence, the performance of the proposed CS artifact suppression method is evaluated using the (log) mean 


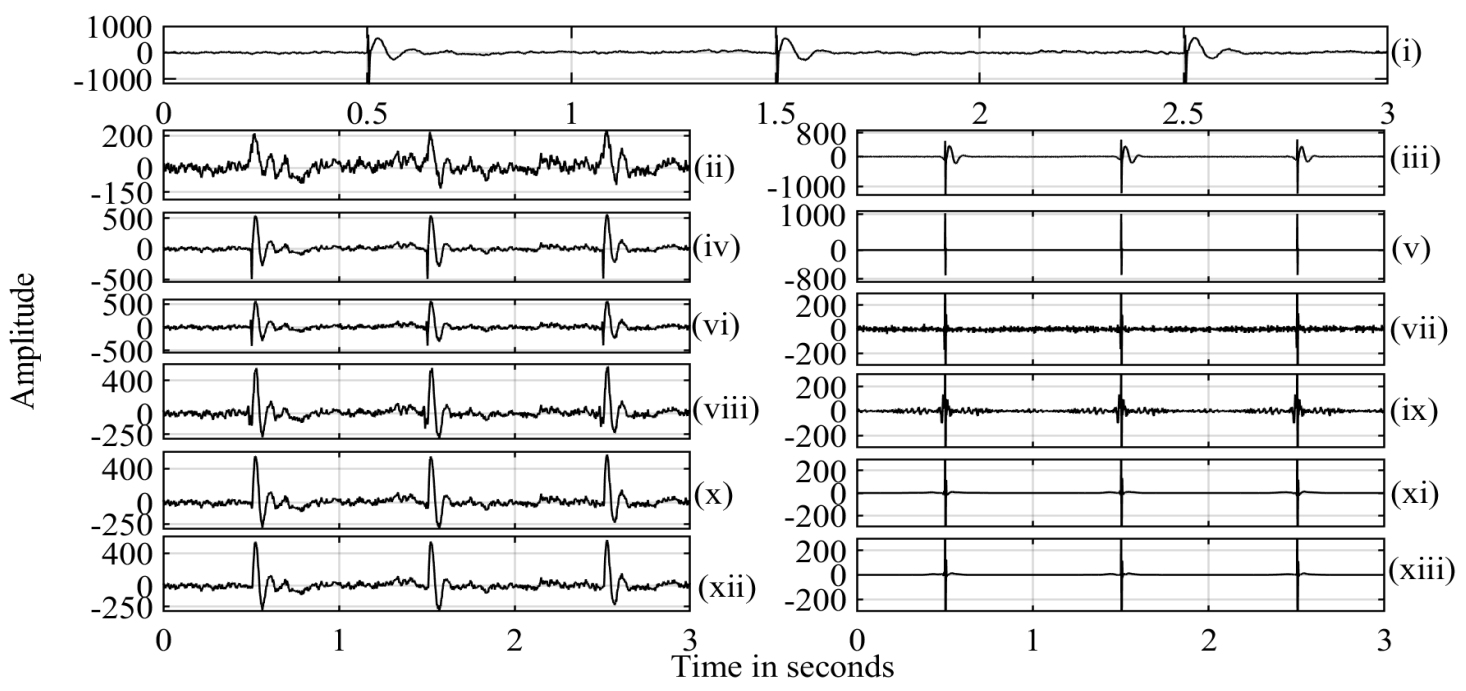

Fig. 8: Plots of (i) monochannel simulated SEEG signal affected by LFCS, (ii)-(iii) physiological background and artefact pattern obtained using classical TQWT (without nonlinear processing), (iv)-(v) physiological background and artefact pattern obtained using model based method [6], (vi)-(vii) physiological background and artefact pattern obtained using FIR-GEVD method [8], (viii)-(ix) physiological background and artefact pattern obtained using Hampel filtering method [7], (x)-(xi) physiological background and artefact pattern obtained using WASOBI-TQWT method, (xii)-(xiii) physiological background and artefact pattern obtained using SCA-TQWT method.

square (MSE) error measure, expressed as:

$$
\mathrm{MSE}=\log \left(\frac{1}{C} \sum_{i=1}^{C} \frac{1}{N} \sum_{n=1}^{N}\left(x_{i}(n)-x_{i}^{\mathrm{est}}(n)\right)^{2}\right)
$$

where $C$ and $N$ denote the number of channels and total number of samples respectively. $x_{i}(n)$ and $x_{i}^{\text {est }}(n)$ represent ground truth $i^{\text {th }}$ channel signal and its estimate, respectively.

However, in case of real SEEG signals, the original clean sources are unknown. Thus, we compute the correlation coefficient between the estimated clean EEG signal with the synthetic CS artifact pattern. Firstly, the correlation coefficient is computed between estimated $i^{\text {th }}$ sensor signal $x_{i}^{\text {est }}(n)$ and the theoretical CS artifact pattern $p(n)(1)$ :

$$
\rho_{i}(m)=\frac{\operatorname{COV}\left(x_{i}^{\text {est }}(n) p(n-m)\right)}{\sigma_{x_{i}^{\text {est }}(n)} \sigma_{p}}
$$

where COV represents covariance operation. 


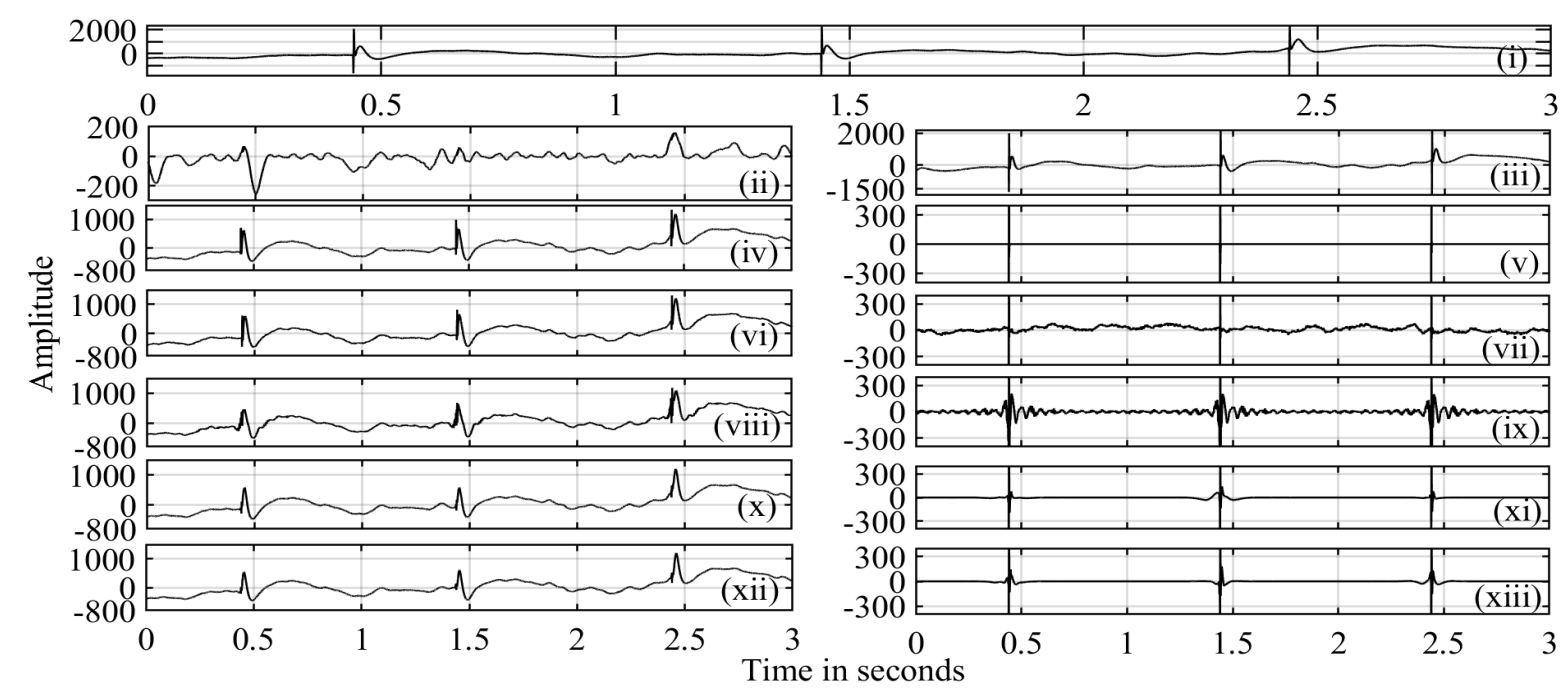

Fig. 9: Plots of (i) monochannel real SEEG signal affected by LFCS, (ii)-(iii) physiological background and artefact pattern obtained using classical TQWT (without nonlinear processing), (iv)-(v) physiological background and artefact pattern obtained using model based method [6], (vi)-(vii) physiological background and artefact pattern obtained using FIR-GEVD method [8], (viii)-(ix) physiological background and artefact pattern obtained using Hampel filtering method [7], (x)-(xi) physiological background and artefact pattern obtained using WASOBI-TQWT method, (xii)-(xiii) physiological background and artefact pattern obtained using SCA-TQWT method.

Finally, the correlation coefficient index (CCI) is computed as,

$$
C C I=\frac{100}{C} \sum_{i=1}^{C} \max _{m}\left|\rho_{i}(m)\right|
$$

where $C$ is the number of SEEG channels.

\section{RESULTS AND DISCUSSION}

In this section we present the performance of the proposed time-frequency-spatial filtering methods in suppressing multichannel LFCS and HFCS artifacts, both on simulated and real signals (see Figs. 2 and 3 for examples of LFCS recordings). 
The proposed combinations of spatial filtering and TQWT were compared with three existing CS artefact suppression methods namely FIR-GEVD method [8] ${ }^{2}$, frequency domain Hampel filtering method [7], and model based artefact correction method [6]. In [7], Hampel identifier finds the spectral outliers (harmonics generated due to CS artifacts) in the real and imaginary spectrum and replaces them with interpolated values [7]. However as discussed in [7], the entire FFT spectrum is not contaminated with CS artefacts (especially the low frequency region of the spectrum). Therefore, we have corrected the outliers in FFT spectrum which appeared above $20 \mathrm{~Hz}$ using Hampel filtering while keeping the low frequency bins of the spectrum unaltered. For implementation of model based CS artefact correction method, various artefact shapes have been obtained from the output of the RC circuit (models electrode-tissue interface) for different RC time constants as described in [6]. However, the results obtained for HFCS artifact correction using model based method are not very satisfactory (note that the method was proposed for LFCS artifact correction) as most of the time the corrected signal contained high artifact residuals. Moreover for HFCS, as the artifacts appear very frequently, it becomes computationally cumbersome to compare every artifact from each channel with a very large dictionary for the best matching. Therefore, we do not present the numerical results of HFCS artifact correction using model based method. We have also compared our combined methodology with a channel by channel TQWT approach, but the quantitative results for this comparison will not be presented, as they are both less convincing and they require a much higher computational burden.

\section{A. Synthetic signals}

Table I presents the mean and standard deviation (std) of MSE values (for 50 different sessions) obtained using different methods on synthetic multichannel SEEG signals. More precisely, we compare two spatial filtering - TQWT based methods (SCA-TQWT and BSS-TQWT) with existing FIR-GEVD [8], Hampel filtering [7], and model based methods (for LFCS only) [6]. For LFCS, we have obtained least MSE values using SCA-TQWT method followed by BSSTQWT (the best performances were obtained for WASOBI [40]), Hampel filtering method, model based method, and FIR-GEVD method. For HFCS, WASOBI-TQWT seems to perform slightly

\footnotetext{
${ }^{2}$ In [8], the results corresponding to different filters were similar. Thus, we have tested the FIR-GEVD approach only. Concerning the number of artifact sources, we have followed the approach given by [8], i.e., we have selected the two last components.
} 
TABLE I: Obtained MSE values corresponding to proposed methods and existing methods in suppressing CS artifacts of synthetic SEEG signals (mean \pm std).

\begin{tabular}{|c|c|c|c|c|c|c|}
\hline $\begin{array}{c}\text { Stimulation } \\
\text { type }\end{array}$ & $\begin{array}{c}\text { Raw } \\
\text { SEEG }\end{array}$ & $\begin{array}{c}\text { SCA- } \\
\text { TQWT }\end{array}$ & $\begin{array}{c}\text { WASOBI- } \\
\text { TQWT }\end{array}$ & $\begin{array}{c}\text { FIR- } \\
\text { GEVD [8] }\end{array}$ & $\begin{array}{c}\text { Hampel } \\
\text { filtering [7] }\end{array}$ & $\begin{array}{c}\text { Model based } \\
\text { method [6] }\end{array}$ \\
\hline LFCS & $3.52 \pm 0.05$ & $1.49 \pm 0.24$ & $1.83 \pm 0.22$ & $3.17 \pm 0.19$ & $2.51 \pm 0.12$ & $2.87 \pm 0.05$ \\
\hline HFCS & $3.22 \pm 0.11$ & $2.12 \pm 0.12$ & $2.11 \pm 0.13$ & $2.99 \pm 0.15$ & $2.47 \pm 0.12$ & NR \\
\hline
\end{tabular}

TABLE II: Obtained CCI values for proposed methods and existing methods in suppressing CS artifacts of real SEEG signals.(mean \pm std).

\begin{tabular}{|c|c|c|c|c|c|c|}
\hline $\begin{array}{c}\text { Stimulation } \\
\text { type }\end{array}$ & $\begin{array}{c}\text { Raw } \\
\text { SEEG }\end{array}$ & $\begin{array}{c}\text { SCA- } \\
\text { TQWT }\end{array}$ & $\begin{array}{c}\text { WASOBI- } \\
\text { TQWT }\end{array}$ & $\begin{array}{c}\text { FIR- } \\
\text { GEVD [8] }\end{array}$ & $\begin{array}{c}\text { Hampel } \\
\text { filtering [7] }\end{array}$ & $\begin{array}{c}\text { Model based } \\
\text { method [6] }\end{array}$ \\
\hline LFCS & $3.39 \pm 1.85$ & $0.16 \pm 0.06$ & $0.34 \pm 0.12$ & $0.93 \pm 0.51$ & $0.84 \pm 0.35$ & $1.07 \pm 0.75$ \\
\hline HFCS & $3.78 \pm 2.49$ & $0.30 \pm 0.19$ & $0.60 \pm 0.41$ & $1.27 \pm 1.07$ & $1.18 \pm 1.02$ & NR \\
\hline
\end{tabular}

better than SCA-TQWT, but the results are hardly different and the task of artifacted component selection is more difficult and more subjective for the BSS based methods than for the SCA (for the latter, the components similar to the dictionary appear in the common subspace, while for the BSS, the source order is randomly permuted [23]).

Fig. 8 illustrates the results on an example of simulated signals. For readability, only one channel is presented. One can notice that the classical TQWT identify as transient the CCEP, as well as other short-duration events, while FIR-GEVD and Hampel filtering leaves a significant part of background activity in the transient components. The model based method fails to remove the CS artifact completely and significant residues of the artifacts still remain in the corrected signal. Our proposed spatial-filtering-TQWT pipeline yields the best results (for this example, MSE values are equal to $3.56,2.79,2.92,2.53,1.43$, and 1.05, respectively for TQWT only, model based method, FIR-GEVD, Hampel filtering, WASOBI-TQWT, and SCA-TQWT).

\section{B. Real signals}

Table II presents the computed mean and std of the CCI values (over 50 different real sessions of stimulations) for the artifact affected SEEG signals, artifact corrected SEEG signals using our 
proposed methods, existing model based method (for LFCS only), Hampel filtering method, and FIR-GEVD method. As expected, the highest CCI values correspond to the artifacted SEEG signal for both LFCS and HFCS artifacts. For these real signals, both LFCS and HFCS, the smallest CCI values are obtained using SCA-TQWT method, followed by WASOBI-TQWT method and existing methods.

Fig. 9 presents a monochannel real SEEG signal with LFCS artefact, the extracted physiological background signals and LFCS artefact patterns using classical TQWT, existing model based method [6], FIR-GEVD [8], Hampel filtering method [7], proposed WASOBI-TQWT, and SCA-TQWT methods. It can be noticed that the proposed methods extract the CS artifact pattern without any physiological background and are more effective to suppress the CS artifacts in real SEEG signals (for this example, CCI values are equal to $0.10,1.32,1.25,1.06,0.48$, and 0.36 respectively for TQWT only, model based method, FIR-GEVD, Hampel filtering, WASOBITQWT, and SCA-TQWT).

\section{Discussion}

The proposed pipeline strengthen the capacities of both TQWT and spatial filtering methods to separate between (transient) artifacts and physiological background. The role of each of the approaches can be illustrated by analyzing the multichannel data. For example, Fig. 3 presents a multichannel SEEG signal subjected to LFCS, whereas Fig. 10 presents the first 15 components after rotation of the multi-channel SEEG signals onto the dictionary using SCA. Fig. 11 presents the obtained clean SEEG signals after applying SCA-TQWT based filtering on the multi-channel SEEG signals (shown in Fig. 3) affected with LFCS artefact. For HFCS, we have presented the multichannel artefacted SEEG signals and the separated 15 sources (using WASOBI) in Figs. 12 and 13, respectively. Fig. 14 shows the recovered clean SEEG signals after applying WASOBITQWT based filtering on the multi-channel SEEG signals (shown in Fig. 12) affected with HFCS artefact.

Analyzing these figures, it appears clearly that it is more convenient to suppress the artifacts in those few components (sources) which mostly represent the CS. Fig. 15 graphically presents the variation of MSE and CCI values with respect to number of cleaned components for both LFCS and HFCS in synthetic and real EEG signals. It can be observed from these figures that maximum number of six cleaned components provided consistent results (least MSE and CCI values) across all data sets. 


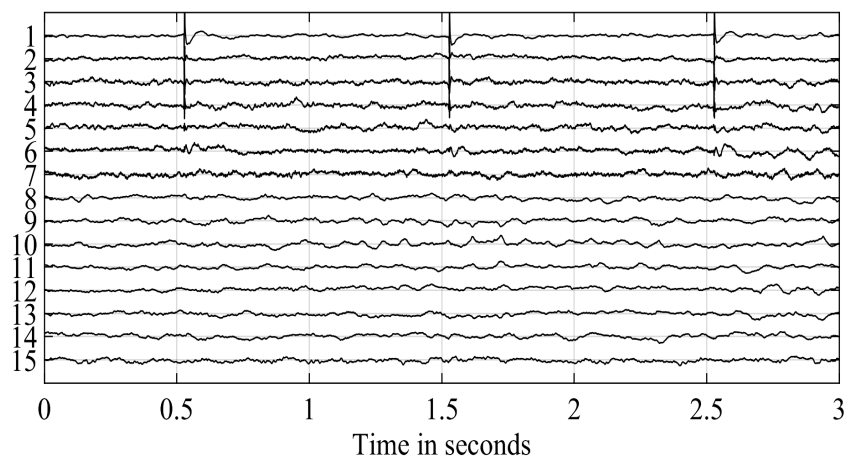

Fig. 10: Plot of first 15 SCA components of the real SEEG signal (shown in Fig. 3 ) affected with LFCS.

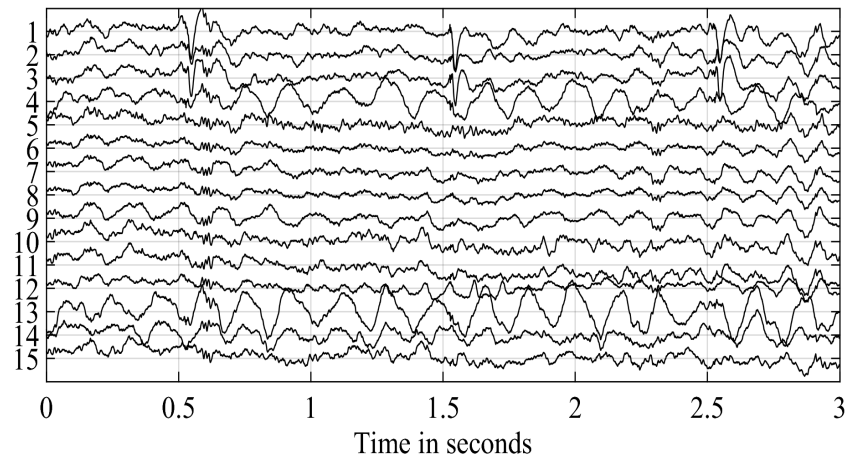

Fig. 11: Plot of obtained artefact free version of the real multi-channel SEEG signal affected with LFCS (shown in Fig. 3) using SCA-TQWT.

Fig. 16 presents the mean spectrum magnitude of a single session before (shown in black) and after (shown in red) suppression of CS artifacts subject to low and high frequency CS. As it can be seen that, the harmonics corresponding to CS artifacts are suppressed in the frequency domain while retaining the desired SEEG signal spectrum.

The key point is to (spatially) separate as correctly as possible between artifacts and physiological signals. Both SCA and BSS methods are found useful in separating the artifact sources from the multichannel SEEG signals. But, while BSS is a generic family of methods based on statistical independence (decorrelation) between artifact physiological sources, SCA explicitly optimizes the separation in order to fit the artifact components onto a predefined dictionary. In theory then, the performances should be better, but they are clearly dependent on the dictionary 
construction (although one can construct, as we did in this paper, a rich dictionary including different possible versions of the artifact, that by linear combination might be able to reproduce the actual recorded ones). On the other hand, although BSS uses less assumptions on the artifacts (no physical model), it uses difficult to verify assumptions (especially the independence between artifacts and physiological sources), which might be even contradicted in practice (as in the CCEP case).

Previous arguments aim to explain the good performances and the robustness of our proposed methods, compared with the literature. Indeed, it has been found that the performance of FIRGEVD method [8] is dependent on the decomposition quality and on the number of components selected as artifactual. For example, the results heavily degrade in the presence of sampling jitters, as the CS artifacts spread over more number of components in the transformed domain after GEVD operation. Moreover, some significant background activity (especially high-frequency) might be found in the selected artifact sources after GEVD, and this activity will be lost after reconstruction.

The model based artifact correction method [6] is on the other hand dependent on finding the best match of the real artifact shape in the constructed dictionary, so it depends on the model of the instrumentation. The performance of this method can probably be further improved if one includes in the dictionary better artifact models or even some real artifact shapes (obtained e.g., by averaging or chosen from the output artifact patterns extracted using other methods). Although our method is also based on modeling the artifact, we allow more variability in the pattern shape, as it does not need to be correlated with a particular element of the dictionary but with some linear combination (i.e., with the dictionary subspace).

Finally, the frequency domain Hampel filtering method is based on the identification of outliers (i.e., high amplitudes) in the real and imaginary part of the spectrum. In the HFCS case, the artifact components are quite easy to identify (they are strong and widely spaced in frequency, with harmonics at avery $50-55 \mathrm{~Hz}$, see [8]), but some of them are aliased in the low frequencies and they will not be seen as outliers. On the other hand, for the LFCS case, the spectrum is much denser in frequency (harmonics spaced by $1 \mathrm{~Hz}$ ) and it also has components in the low frequency part of the spectrum, where physiological activities lie. Detection of outliers is thus more difficult and is unavoidable to miss artifact harmonics in the lower part of the spectrum. 


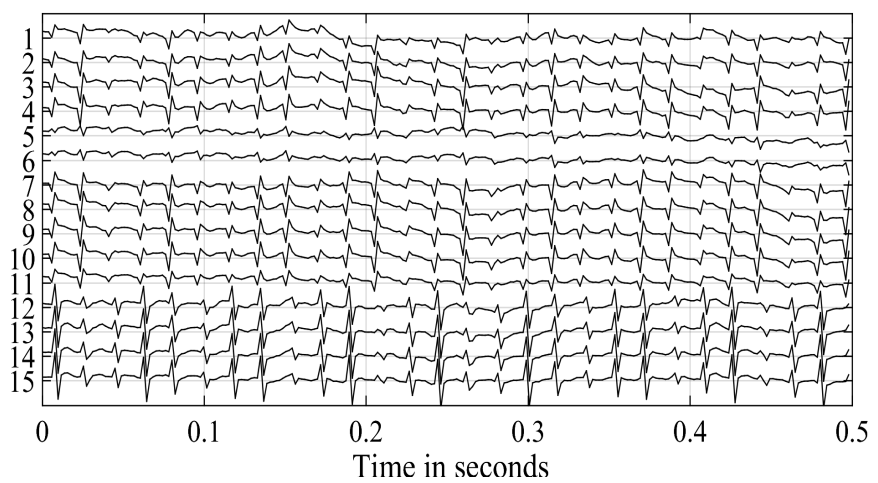

Fig. 12: Real SEEG signals recorded during HFCS with $1 \mathrm{~mA}$ biphasic current pattern.

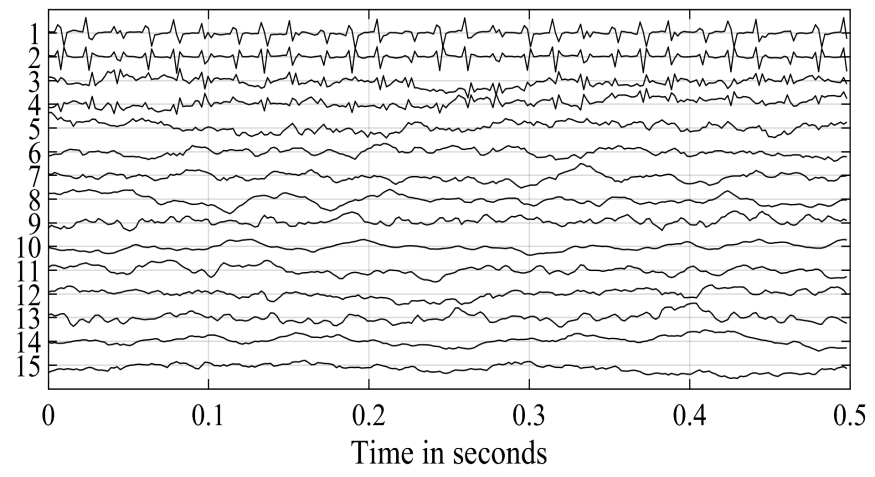

Fig. 13: Plot of 15 WASOBI components of the real SEEG signal (shown in Fig. 12) affected with HFCS.

\section{CONCLUSION AND FUTURE WORK}

We have proposed a family of methods for suppressing cortical stimulation (CS) artifacts from depth multichannel SEEG recordings. The proposed methods are a combination of TQWT based (nonlinear) filtering and different spatial filtering methods (blind source separation and subspace correlation methods). The spatial filtering is optimized to separate the artifacts (on few components/sources, possibly using a dictionary of modeled artifacts) from the physiological signals and it facilitates the task of TQWT, whose aim is to separate a signal (source) into transient and oscillatory parts using dual $Q$ factors. Further, we use known time-frequency information on the CS artifacts to nonlinearly extract the artifact pattern from the remaining oscillatory background in the wavelet domain. 


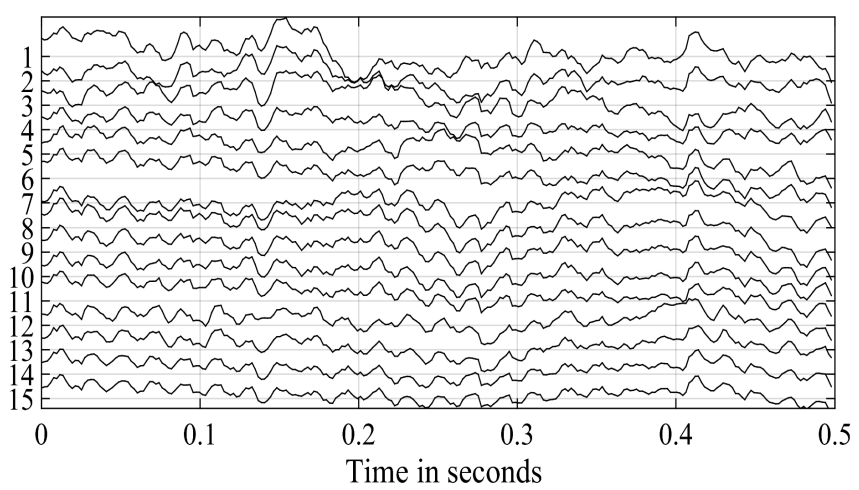

Fig. 14: Plot of obtained artefact free version of the real multi-channel SEEG signal affected with HFCS (shown in Fig. 12) using WASOBI-TQWT.
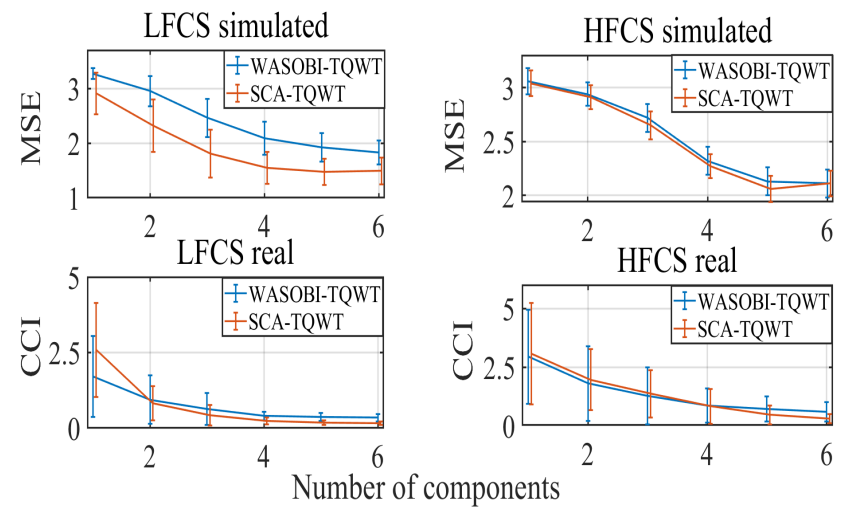

Fig. 15: Plots of the variations of MSE and CCI values with respect to number of cleaned components of synthetic and real multichannel SEEG signals.

The performances of the proposed methods were analyzed using synthetic and real multichannel SEEG signals with low and high frequency artifacts and we compared them with existing mono-channel (Hampel [7] or dictionary based [6]) or multichannel methods (GEVD based [8]). The experimental results clearly indicate the suitability of the proposed methods in suppressing CS artifacts and their robustness to varying acquisition conditions (jitter, noise, ...). Relatively few parameters need to be tuned, the most critical being the choice of the CS artifact sources after spatial filtering (with SCA based spatial filters easier to tune than BSS ones).

Other CS artifact suppression methods could be proposed, using for example adaptive signal decomposition methods like multivariate EMD (MEMD) and its different versions. Our first 


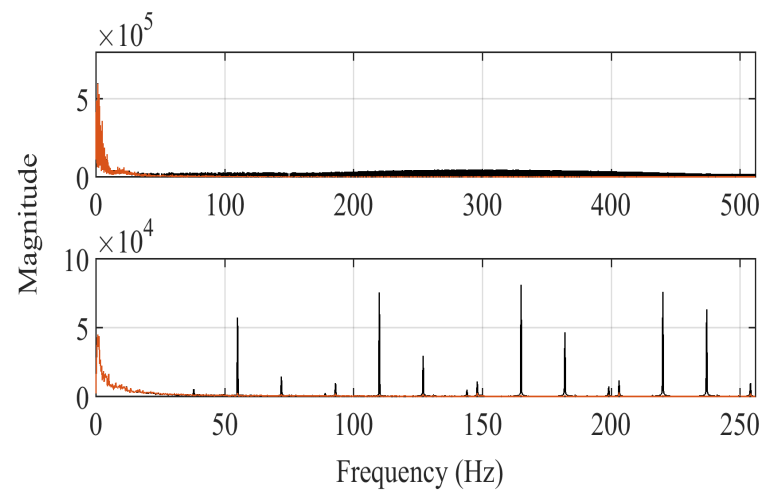

Fig. 16: Plots of the mean spectrum magnitude of a single session before (black) and after (red) applying WASOBI-TQWT method (i) for LFCS (ii) for HFCS. Spectral peaks corresponding to artifacts are absent after processing.

preliminary experiments (see supplementary material) indicate that MEMD method could provide equivalent performances in removing LFCS artifacts from real SEEG signals. However, for HFCS artifacted SEEG, the reconstructed signal obtained using MEMD does not properly preserve high frequency contents of the signal. Finally, noise-assisted MEMD (NA-MEMD) and other improved multivariate signal decomposition methods [9] could be tested in the future for CS artifact separation.

\section{ACKNOWLEDGEMENT}

This work was carried out during the visit of first author at the Université de Lorraine, CNRS, CRAN, F-54000 Nancy, France under Raman-Charpak fellowship 2017. The authors would like to thank Indo-French Centre for the Promotion of Advanced Research (IFCPAR) for their support during this work.

\section{REFERENCES}

[1] S. Kovac et al., "Seizures induced by direct electrical cortical stimulation-Mechanisms and clinical considerations," Clinical Neurophysiology, vol. 127, no. 1, pp. 31-39, 2016.

[2] M. Gavaret et al., "Source localization of scalp-EEG interictal spikes in posterior cortex epilepsies investigated by HR-EEG and SEEG,” Epilepsia, vol. 50, no. 2, pp. 276-289, 2009.

[3] L. Koessler et al., "Source localization of ictal epileptic activity investigated by high resolution EEG and validated by SEEG," Neuroimage, vol. 51, no. 2, pp. 642-653, 2010. 
[4] J. Bancaud et al., ““Generalized” epileptic seizures elicited by electrical stimulation of the frontal lobe in man," Electroencephalography and Clinical Neurophysiology, vol. 37, no. 3, pp. 275-282, 1974.

[5] A. Zangaladze et al., "The effectiveness of low-frequency stimulation for mapping cortical function," Epilepsia, vol. 49, no. 3, pp. 481-487, 2008.

[6] L. Trebaul et al., "Stimulation artifact correction method for estimation of early cortico-cortical evoked potentials," Journal of Neuroscience Methods, vol. 264, pp. 94-102, 2016.

[7] D. P. Allen et al., "Suppression of deep brain stimulation artifacts from the electroencephalogram by frequency-domain Hampel filtering," Clinical Neurophysiology, vol. 121, no. 8, pp. 1227-1232, 2010.

[8] J. Hofmanis et al., "Denoising depth EEG signals during DBS using filtering and subspace decomposition," IEEE Transactions on Biomedical Engineering, vol. 60, no. 10, pp. 2686-2695, 2013.

[9] L. Stanković et al., "Time-frequency decomposition of multivariate multicomponent signals," Signal Processing, vol. 142, pp. 468-479, 2018.

[10] A. Bhattacharyya and R. B. Pachori, "A multivariate approach for patient-specific EEG seizure detection using empirical wavelet transform," IEEE Transactions on Biomedical Engineering, vol. 64, no. 9, pp. 2003-2015, 2017.

[11] N. E. Huang et al., "The empirical mode decomposition and the Hilbert spectrum for nonlinear and non-stationary time series analysis," in Proceedings of the Royal Society of London A: mathematical, physical and engineering sciences, vol. 454, no. 1971, 1998, pp. 903-995.

[12] I. Daubechies et al., "Synchrosqueezed wavelet transforms: An empirical mode decomposition-like tool," Applied and computational harmonic analysis, vol. 30, no. 2, pp. 243-261, 2011.

[13] N. Rehman and D. P. Mandic, "Multivariate empirical mode decomposition," in Proceedings of The Royal Society of London A: Mathematical, Physical and Engineering Sciences, 2009, p. rspa20090502.

[14] N. Ur Rehman and D. P. Mandic, "Filter bank property of multivariate empirical mode decomposition," IEEE Transactions on Signal Processing, vol. 59, no. 5, pp. 2421-2426, 2011.

[15] A. Ahrabian et al., "Synchrosqueezing-based time-frequency analysis of multivariate data," Signal Processing, vol. 106, pp. 331-341, 2015.

[16] D. P. Mandic et al., "Empirical mode decomposition-based time-frequency analysis of multivariate signals: The power of adaptive data analysis," IEEE Signal Processing Magazine, vol. 30, no. 6, pp. 74-86, 2013.

[17] C. Park et al., "Classification of motor imagery BCI using multivariate empirical mode decomposition," IEEE Transactions on Neural Systems and Rehabilitation Engineering, vol. 21, no. 1, pp. 10-22, 2013.

[18] M. Hu and H. Liang, "Adaptive multiscale entropy analysis of multivariate neural data," IEEE Transactions on Biomedical Engineering, vol. 59, no. 1, pp. 12-15, 2012.

[19] I. W. Selesnick, "Wavelet transform with tunable Q-factor," IEEE Transactions on Signal Processing, vol. 59, no. 8, pp. 3560-3575, 2011.

[20] I. W. Selesnick, "Sparse signal representations using the tunable Q-factor wavelet transform," Wavelets and Sparsity XIV, vol. 8138, p. 81381U, 2011.

[21] S. Patidar and R. B. Pachori, "Segmentation of cardiac sound signals by removing murmurs using constrained tunable-Q wavelet transform,” Biomedical Signal Processing and Control, vol. 8, no. 6, pp. 559-567, 2013.

[22] A. Bhattacharyya et al., "Tunable-Q wavelet transform based multiscale entropy measure for automated classification of epileptic EEG signals," Applied Sciences, vol. 7, no. 4, p. 385, 2017.

[23] A. Cichocki and S. Amari, Adaptive Blind Signal and Image Processing Learning Algorithms and Applications. John Wiley \& Sons, New York, USA, 2002.

[24] G. H. Golub and C. F. Van Loan, Matrix computations, 2012, vol. 3. 
[25] V. Fonov et al., "Unbiased nonlinear average age-appropriate brain templates from birth to adulthood," NeuroImage, vol. 47, Supplement 1, pp. S102 -, 2009, organization for Human Brain Mapping 2009 Annual Meeting.

[26] . . *, "MNI1552 Head Model, UCL." [Online]. Available: http://www.ucl.ac.uk/medphys/research/borl/resources/ adultMNImodel

[27] V. Caune et al., "Evaluating dipolar source localization feasibility from intracerebral SEEG recordings," Neuroimage, vol. 98, pp. 118-133, 2014.

[28] R. Ranta et al., "Assessing human brain impedance using simultaneous surface and intracerebral recordings," Neuroscience, vol. 343, pp. 411-422, 2017.

[29] L. Rankine et al., "A nonstationary model of newborn EEG," IEEE Transactions on Biomedical Engineering, vol. 54, no. 1, pp. 19-28, 2007.

[30] V. Caune et al., "EEG/SEEG signal modelling using frequency and fractal analysis," in BIOSIGNALS, 2012, pp. $249-253$.

[31] M. V. Afonso et al., "Fast image recovery using variable splitting and constrained optimization," IEEE Transactions on Image Processing, vol. 19, no. 9, pp. 2345-2356, 2010.

[32] I. W. Selesnick, "A new sparsity-enabled signal separation method based on signal resonance," in 2010 IEEE International Conference on Acoustics, Speech and Signal Processing, March 2010, pp. 4150-4153.

[33] R. Romo-Vázquez et al., "Blind source separation, wavelet denoising and discriminant analysis for EEG artefacts and noise cancelling," Biomedical Signal Processing and Control, vol. 7, no. 4, pp. 389 - 400, 2012.

[34] H. Hotelling, "Relations between two sets of variates," Biometrika, vol. 28, no. 3/4, pp. 321-377, 1936.

[35] P. Comon and C. Jutten, Eds., Handbook of Blind Source Separation. Independent Component Analysis and Applications. Elsevier, 2010.

[36] A. Hyvärinen, "Fast and robust fixed-point algorithms for independent component analysis," IEEE Transactions on Neural Networks, vol. 10, no. 3, pp. 626-634, 1999.

[37] T. W. Lee et al., "Independent Component Analysis using an Extended Infomax algorithm for mixed subgaussian and supergaussian sources," Neural Computation, vol. 11, pp. 417-441, 1999.

[38] J. Cardoso, "High-order contrasts for independent component analysis," Neural Computation, vol. 11, no. 1, pp. 157-192, 1999.

[39] A. Belouchrani et al., "A blind source separation technique using second-order statistics," IEEE Transactions on Signal Processing, vol. 45, no. 2, pp. 434-444, 1997.

[40] A. Yeredor, "Blind separation of gaussian sources via second-order statistics with asymptotically optimal weigthting," IEEE Signal Processing Letters, vol. 7, pp. 197-200, Jul 2000.

[41] P. Tichavskỳ et al., "Blind signal separation by combining two ICA algorithms: HOS-based EFICA and time structure-based WASOBI," in Signal Processing Conference, 2006 14th European, 2006, pp. 1-5.

[42] P. Tichavsky et al., "A Hybrid Technique for Blind Non-Gaussian and Time-Correlated Sources Using a Multicomponent Approach,” IEEE Transactions on Neural Networks, vol. 19, no. 3, pp. 421-430, March 2008.

[43] J. Elsner and A. Tsonis, Singular Spectrum Analysis. Springer, 1996.

[44] Z. J. Koles, "The quantitative extraction and topographic mapping of the abnormal components in the clinical eeg," Electroencephalography and clinical Neurophysiology, vol. 79, no. 6, pp. 440-447, 1991.

[45] C. Park et al., "Augmented complex common spatial patterns for classification of noncircular EEG from motor imagery tasks," IEEE Transactions on Neural Systems and Rehabilitation Engineering, vol. 22, no. 1, pp. 1-10, 2014. 